\title{
TRANSFORMATION OF BASES FOR RELATIVE LINEAR SETS*
}

\author{
MARGARETE C. WOLF $†$
}

The definitions of linear independence, dependence, and extension of sets of vectors relative to a matrix which are used in this paper were recently introduced by $M$. H. Ingraham, $\ddagger$ and are not repeated here. The purpose of the present paper is to study the structure of basal elements of a linear extension relative to a matrix. There are developed necessary and sufficient conditions which the elements of a matrix of a transformation must satisfy so that one set of basal elements of a vector space can be transformed into another basal set.

It is assumed throughout this paper that the elements of the matrices and the vectors, as well as the coefficients of the polynomials, are in a field $\mathfrak{F}$. The following theorem is used but stated without proof : $\S$

THEOREM 1. If $\xi_{1}, \xi_{2}, \cdots, \xi_{k}$ and $\eta_{1}, \eta_{2}, \cdots, \eta_{l}$ are two sets of vectors ( $n \times 1$ matrices), each of which is linearly independent relative to an $n \times n$ matrix $M$, such that $L_{M}\left(\xi_{1}, \xi_{2}, \cdots, \xi_{k}\right)=L_{M}\left(\eta_{1}, \eta_{2}, \cdots, \eta_{l}\right)$, if $h_{1 i}$ and $h_{2 i}$ are, respectively, the minimum polynomials associated with $\xi_{i}$ and $\eta_{i}$ relative to $M$, and if $g$ is an irreducible polynomial and $t$ any positive integer, then the number of polynomials $h_{1 i}$ divisible by $g^{t}$ is equal to the number of $h_{2 i}$ divisible by $g^{t}$.

This is essentially the only restriction on the polynomials $h_{1 i}$ and $h_{2 i}$.

THEOREM 2. If $\xi_{1}, \xi_{2}, \cdots, \xi_{k}$ is a proper base relative to a matrix $M$ for the space $L_{M}\left(\xi_{1}, \xi_{2}, \cdots, \xi_{k}\right)$, if the minimum polynomial associated with $\xi_{i}$ relative to $M$ is $g^{t i}$, a power of an irreducible polynomial $g$, and if $\eta_{i}=\sum_{j=1}^{k} f_{i j}(M) \xi_{j},(i=1,2, \cdots, k)$, where the $f_{i j}$ are polynomials with coefficients in the field $\mathfrak{F}$, necessary and sufficient conditions that the $\eta_{i}$ form a proper base relative to $M$ are that the set of polynomials minimally associated with the $\eta_{i}$ is exactly the set $g^{t^{i}}$ in some order, and

* Presented to the Society, December 29, 1937.

$\dagger$ The work of M. C. Wolf was supported by a grant from the Wisconsin Alumni Research Foundation. The topic was suggested by M. H. Ingraham, who also contributed valuable ideas and criticisms when the paper was presented to a seminar.

$\ddagger$ M. H. Ingraham and M. C. Wolf, Relative linear sets and similarity of matrices whose elements belong to a division algebra, Transactions of this Society, vol. 42 (1937), pp. 16-31.

$\S$ M. H. Ingraham and M. C. Wolf, loc. cit. 
that the $k \times k$ matrix $\left(f_{i j}\right)$ is of rank $k$ in the ring of polynomials reduced modulo $g$.

The necessity of the conditions will be proved first. Obviously the minimum polynomial associated with $\eta_{i}$ is a power of $g$; call it $g^{s i}$. From Theorem 1 it follows that the set of integers $t_{i}$ is equal to the set $s_{i}$. Let $\eta$ and $\xi$ be vectors of vectors with $\eta_{i}$ and $\xi_{i}$, respectively, as the $i$ th element. Let $F=\left(f_{i j}\right)$ be the matrix with the polynomial $f_{i j}$ in the $i$ th row and $j$ th column, and $F(M)$ the matrix of matrices $\left(f_{i j}(M)\right)$. The relations $\eta_{i}=\sum_{j=1}^{k} f_{i j}(M) \xi_{j},(i=1,2, \cdots, k)$, can be written as $\eta=F(M) \xi$. A necessary condition that $L_{M}\left(\xi_{1}, \xi_{2}, \cdots, \xi_{k}\right)$ $=L_{M}\left(\eta_{1}, \eta_{2}, \cdots, \eta_{k}\right)$ is that a matrix $G$ exist such that $\xi=G(M) \eta$. Hence it is necessary that $G$ exist such that $\xi=G(M) F(M) \xi$. The matrix $G F$ is square with elements $w_{i j}$, polynomials with coefficients in $\mathfrak{F}$, and $\xi_{i}=\sum_{j=1}^{k} w_{i j}(M) \xi_{j}$. The vectors $\xi_{1}, \xi_{2}, \cdots, \xi_{k}$ are by hypothesis linearly independent relative to $M$; hence $w_{j j} \equiv 1\left(\bmod g^{t_{j}}\right)$, and $w_{i j} \equiv 0\left(\bmod g^{t j}\right),(i \neq j)$. Hence it is necessary that $G F \equiv I(\bmod g)$ and that the determinant $|G F| \equiv 1(\bmod g)$. The matrices $F$ and $G$ are square; hence $|G F|=|G||F| \equiv 1(\bmod g)$. Consequently $|F|=\left|\left(f_{i j}\right)\right|$ $\not \equiv 0(\bmod g)$.

The conditions are sufficient, for if $|F| \not \equiv 0(\bmod g)$, then for any positive integer $t,\left(|F|, g^{t}\right)=1$, and there exists a polynomial denoted by $|F|^{-1}$, such that $|F| \cdot|F|^{-1} \equiv 1\left(\bmod g^{t}\right)$. Let $G=|F|^{-1}$ adj $F$, where $\operatorname{adj} F$ is the adjoint of the matrix $F$. Then $G F \equiv I\left(\bmod g^{t}\right)$. Hence $G(M) F(M) \xi=\xi$; that is, $G(M) \eta=\xi$, and consequently $\eta_{1}, \eta_{2}, \cdots, \eta_{k}$ form a base relative to $M$ for $L_{M}\left(\xi_{1}, \xi_{2}, \cdots, \xi_{k}\right)$. Let $g^{s_{i}}$ be minimally associated relative to $M$ with $\eta_{i}$, where the set of $s_{i},(i=1,2, \cdots, k)$, is equal to the set of $t_{i},(i=1,2, \cdots, k)$, in some order. The proof that these $\eta_{i}$ are linearly independent relative to $M$ is based on the theorem that the number of basal elements in a proper base for a space relative to a field $\mathfrak{F}$ is independent of the base.

From Theorem 1 and from the fact that it is necessary that $\left|\left(f_{i j}\right)\right| \not \equiv 0(\bmod g)$, the $\eta_{i}$ may be rearranged so that the minimum polynomial associated with $\eta_{i}$ is $g^{t_{i}}$, where $t_{1} \geqq t_{2} \geqq \cdots \geqq t_{k}$. Then $\eta_{i}$ is of the form

$$
\begin{aligned}
\eta_{i}= & g^{t_{1}-t_{i}}(M) h_{i 1}(M) \xi_{1}+g^{t_{2}-t_{i}}(M) h_{i 2}(M) \xi_{2}+\cdots \\
& +g^{t_{i-1}-t_{i}}(M) h_{i i-1}(M) \xi_{i-1} \\
& +f_{i i}(M) \xi_{i}+f_{i i+1}(M) \xi_{i+1}+\cdots+f_{i k}(M) \xi_{k}
\end{aligned}
$$

where $\left(f_{i i}, g\right)=1$. If $t_{i}>t_{i+1}$, the $h_{i j}$ and the $f_{i j}$ are arbitrary polynomials. However if $t_{i-s-1}>t_{i-s}=t_{i-s+1}=\cdots=t_{i}>t_{i+1}$, then the prin- 
cipal minor determinant with diagonal elements $f_{i-s} i_{-s}, \cdots, f_{i-1}{ }_{i-1}, f_{i i}$ cannot be congruent to zero modulo $g$. Obviously, if the vectors $\eta_{i}$ are of the form (1), and if the above conditions on the $h_{i j}$ and $f_{i j}$ are satisfied, the sufficient conditions of Theorem 2 are fulfilled.

TheORem 3. If the set of vectors $\xi_{1}, \xi_{2}, \cdots, \xi_{r}$ is a proper base relative to a matrix $M$ for $L_{M}\left(\xi_{1}, \xi_{2}, \cdots, \xi_{r}\right)$, if $\eta_{k}=\sum_{j=1}^{r} f_{k j}(M) \xi_{j}$, where the $f_{k j}$ are polynomials with coefficients in $\mathfrak{F}$, and if $\prod_{i=1}^{u} g_{i}{ }^{t i i}$ and $\prod_{i=1}^{v} g_{i}{ }^{s_{k i}}$ are minimally associated with $\xi_{j}$ and $\eta_{k}$, respectively, where the $g_{i}$ are polynomials irreducible in $\mathfrak{F}$, necessary and sufficient conditions that the $\eta_{k}$ form a proper base for $L_{M}\left(\xi_{1}, \xi_{2}, \cdots, \xi_{r}\right)$ are: (i) for every $i$ the set of non-zero integers $s_{k i}$ is equal to the set of non-zero $t_{j i}$ in some order, and (ii) for every $i$ and for those $v_{i}$ values of $j$ for which $g_{i}$ is a factor of the minimum polynomial associated with $\xi_{j}$, the $v_{i}$ columns of $\left(f_{k j}\right)$ are of rank $v_{i}$ in the ring of polynomials reduced modulo $g_{i}$.

Let exactly $v_{i}$ of the polynomials minimally associated with the $\xi_{j}$ have a factor $g_{i}$. Then as a consequence of Theorem 1 exactly $v_{i}$ of the polynomials minimally associated with the $\eta_{k}$ have a factor $g_{i}$. Let $p_{i}=\prod_{k \neq i} g_{k}^{c_{k}}$, where for every value of $k$ the $c_{k}$ is the maximum value of $t_{j k},(j=1,2, \cdots, r)$.

After a rearrangement of the $\xi_{j}$ and $\eta_{k}$ it may be assumed that $p_{i}(M) \xi_{j}=p_{i}(M) \eta_{j}=0,\left(j \geqq v_{i}+1\right)$, and that $p_{i}(M) \xi_{j} \neq 0, p_{i}(M) \eta_{j} \neq 0$, $\left(j<v_{i}\right)$. If polynomials exist such that $\xi_{j}=\sum_{k} q_{j k}(M) \eta_{k}$, then

$$
p_{i}(M) \xi_{j}=\sum_{k=1}^{v_{i}} q_{j k}(M) p_{i}(M) \eta_{k} .
$$

The non-zero $p_{i}(M) \xi_{j}$ are linearly independent relative to $M$ for every $i$. Therefore by Theorem 2 for $k, j=1,2, \cdots, v_{i},\left(f_{k j}\right)$ is of rank $v_{i}$ in the ring of polynomials reduced modulo $g_{i}$. If $j \geqq v_{i}+1$, then $f_{j k} \equiv 0\left(\bmod g_{i}{ }^{t_{k i}}\right)$ for $k=1,2, \cdots, v_{i}$, since $\left(p_{i}, g_{i}\right)=1$ and since $p_{i}(M) \eta_{j}=0$ when $j \geqq v_{i}+1$. For different values of $i$ those $v_{i}$ columns, which are of rank $v_{i}$ in the ring of polynomials reduced modulo $g_{i}$, may overlap.

That the conditions of the theorem are sufficient for a proper base follows in a similar manner, since a greatest common divisor of the $p_{i}$ is 1 .

The problem of the transformation of bases of relative linear sets is being studied also in the case where $\mathfrak{F}$ is a division algebra, not necessarily commutative.

The University of Wisconsin 